\title{
Microwave Aquametry-Needs and Perspectives
}

\author{
Andrzej W. Kraszewski, Member, IEEE
}

\begin{abstract}
Determination of moisture content or water content in materials with microwave radiation has several advantages over other electrical methods applying lower frequencies. Problems concerning the development of microwave equipment for those purposes are reviewed. Trends of further development of the instrumentation and research concepts are considered.
\end{abstract}

\section{INTRODUCTION}

A $S$ the application of microwaves in telecommunication and radar systems, both military and civilian, faces limitations and cutbacks, the attention of manufacturers and designers shifts into new fields of potential application. Industrial and scientific applications have been developing at a slow pace since World War II, mainly in two areas: material processing and the monitoring of electrical and nonelectrical quantities. The most popular application of microwave power has been the microwave oven for domestic and commercial cooking. This market is well established and growing. Much less has been invested in industrial microwave power equipment, in spite of the demonstration of a great variety of applications in such industries as chemicals, food, ceramics, rubber, plastics, lumber, tobacco, oil, leather, and pharmaceuticals. The main advantages in using microwave power for processing materials are increased rate of production, improved product characteristics, uniform processing, lower floor-space requirements, convenience, and control over the process. The main disadvantages are a relatively high initial investment and higher operating costs. Sometimes other factors, not necessarily valid but perceived as being so by the industry, have impeded general acceptance of microwave power in industrial processing. These factors include unjustified concern about possible health hazards, lack of application knowledge, and lack of standard designs [1], [2]. Microwave-generated heat has also been used for therapeutic purposes in diathermy for many years. More recently, electromagnetically induced local hyperthermia has gained serious clinical acceptance in cancer treatment.

Low-intensity microwave radiation has found industrial and scientific application in noninvasive and nondestructive testing and monitoring of materials, objects, and

Manuscript received July 24, 1990; revised October 22, 1990.

The author is with the Richard B. Russel Agricultural Research Center, Agricultural Research Service, U.S. Department of Agriculture, Athens, GA 30613.

IEEE Log Number 9143003. persons. Examples of industrial applications include continuous moisture content monitoring and control; monitoring of velocity (police traffic radars, and radar detectors), acceleration, geometrical dimensions, and distance (displacement and level meters); and railway car identification systems [1]. In medical diagnosis, microwave methods have been used for cancer detection and monitoring of pulmonary edema and cardiovascular and respiratory systems. Of all these applications, moisture content determination has the longest and most successful history and the most promising perspectives, as discussed in [2]-[4]. The main obstacles to broader application of microwave instrumentation have included the following: high cost, large equipment dimensions, often inappropriate design, inadequate quality of the instruments for industrial applications, and expensive and time-consuming preparation of measuring sensors [5]. Most of those obstacles can be overcome today with the existing state of microwave technology, the manufacturing potential of this technology, and human resources.

The objectives of this paper are 1) to review the technical and economic importance of microwave techniques for moisture content determination in industrial processes, 2 ) to point out certain areas that require more consideration, and 3) to list the most urgent problems to be solved.

\section{Basic Definitions and Principles}

The moisture content of material, $M$, expressed in percentage, wet basis, is defined as

$$
M=\frac{w_{w}}{w_{w}+w_{d}} \times 100=100 m
$$

that is, as the ratio of the mass of water, $w_{w}$, to the total mass of wet material, where $w_{d}$ is the mass of dry material, and $m$ is the fractional moisture content, wet basis. Because the moisture content is a concept related to a certain volume of material, $v$, (1) may be rewritten in the form

$$
m=\frac{\frac{w_{w}}{v}}{\frac{w_{w}}{v}+\frac{w_{d}}{v}}=\frac{k}{\rho}
$$


where $k$ is the water concentration in the unit volume of material and $\rho$ is the density of the moist material.

Changes of an electrical signal, regardless of operating frequency, interacting with a moist material are proportional to the water concentration, $k$, and are affected only to a small extent by the mass of dry material, $w_{d}$. Thus, when $k$ can be determined from electrical measurement, it is evident from (2) that determination of moisture content, $m$, requires the density of the wet material, $\rho$, to be known. This information can be obtained from a separate density measurement, for example, by weighing a sample of given volume or by using a $\gamma$-ray density gauge. Fluctuations in the density of test material produce effects similar to changes in water content and therefore create a measurement error. This error can be eliminated or limited only if the mass of wet material in the measuring space is held constant during the calibration procedure and during the measurement. For this reason, most existing electrical moisture meters require a sample of strictly determined weight (mass).

Performing fast and accurate moisture content measurements if of great importance in the production, trade, storage, and processing of most products and natural raw materials. Most standard methods of moisture content determination, based on the definition of (1), require weighing a sample, drying it for several hours (up to three days), and reweighing. They provide an accuracy of a few tenths of $1 \%$. For rapid moisture determination, indirect methods of measurement, calibrated against standard methods, have been proposed, tested, introduced into everyday practice, and used for many years. Among these methods, those based on the strong correlation between moisture content and electrical properties of material play an important role [6]. Historically, there has been a trend in the development of instrumentation for moisture content measurement to higher and higher frequencies, from direct current (dc) to alternating current (ac), to radio frequencies ( $R F$ ), and, recently, to microwave frequencies.

This trend is a result of developing opportunities in instrumentation design and manufacturing (microwave integrated circuits, solid-state microwave electronics, CAD for microwave devices, components and systems, etc.), on one hand, and the advantages of microwave measurements of solid and liquid materials containing water, on the other. The natural advantages of microwave methods can be summarized as follows:

- The effect of ionic conductivity of the material decreases with increasing frequency, so it is much smaller than at lower radio frequencies.

- Physical contact between the equipment and the material under test is not required, which makes on-line continuous and remote measurements possible.

- Microwave radiation does not alter or contaminate the test material, as do some of the chemical methods; thus, the measurement is nondestructive.

- Microwave radiation propagates through opaque nonmetallic media, so the volume moisture content can be measured, in contrast to infrared radiation, which is absorbed near the surface.

- Microwave methods are fast and safe, in contrast to ionizing radiation methods.

- Microwave radiation is relatively insensitive to environmental conditions; thus, dust and water vapor do not affect the measurement, in contrast to infrared methods.

General principles and instrumentation for moisture content determination by microwave methods have been discussed previously [5], and recent studies in this area are listed and reviewed in [7] and [8]. For distinguishing moisture measurement from hygrometry, which is a branch of metrology devoted to measurement of water vapor content in gases, the term aquametry has been proposed for the metrological procedures dealing with water content determination in solids and liquids. Microwave aquametry can be defined as a branch of metrology that investigates solids and liquids containing water by identifying their properties in the microwave fields. Microwave aquametry utilizes some well-known physical theories, for example, the theory of dielectric mixtures and the theory of bound water. Beside these cognitive purposes, microwave aquametry has also strictly defined practical objectives. These include the quantitative measurement of water content in materials important from an economic point of view. Since water appears in many natural materials, these objectives have far-reaching economic importance. As an example, drying processes are extremely energy consuming, and continuous moisture content monitoring has become essential. Overdrying not only is costly; in many instances it leads to deterioration in product quality, especially in food and other agricultural products. Thus, precise and real-time automatic control of moisture content is required in many processes, and often the microwave method is the only feasible solution.

The need for controlling the density of wet material during moisture content determination by indirect methods results from the definition of moisture content (eq. (2)). The density of granular material depends upon particle shape and dimensions, temperature, moisture content, and surface structure and condition. Thus, providing a constant material density during continuous moisture content measurement under industrial conditions is a difficult task. Various ways of limiting variations in density have been discussed and tested [5], [9]. It was concluded that the only reliable solution is the use of some densityindependent function, e.g., a relationship between electrical properties of material and its moisture content.

The dispersion and dissipation of electromagnetic energy interacting with dielectric material depend upon the dimensions, shape, and relative permittivity (dielectric properties) of the material. When the moisture content of the material changes, a change is reflected in the wave parameters. Because the relative permittivity of water differs significantly from that of most hygroscopic dielec- 
tric materials, its effect can be separated from the effect of the dry dielectric material. In general, this may be expressed in a functional form [10] as

$$
\alpha=\Phi_{1}\left(w_{w}, w_{d}\right) \text { and } \beta=\Phi_{2}\left(w_{w}, w_{d}\right)
$$

where $\alpha$ and $\beta$ are any two descriptive electromagnetic wave parameters. Regardless of the complexity of the analytical expressions described by (3), it is generally possible to solve the two equations by separation of variables and to express the mass of water and the mass of dry material in terms of two measured parameters in the form

$$
w_{w}=\Psi_{1}(\alpha, \beta) \text { and } w_{d}=\Psi_{2}(\alpha, \beta) .
$$

Substituting the analytical expressions corresponding to (4) into (1), the general expression for moisture content of material can be written as

$$
M=\frac{\Psi_{1}(\alpha, \beta)}{\Psi_{1}(\alpha, \beta)+\Psi_{2}(\alpha, \beta)} \times 100
$$

which contains only the wave parameters determined experimentally and is totally independent of the material density.

\section{INSTRUMENTATION}

Instrumentation for microwave aquametry can be divided into the following groups:

- Portable field meters of very simple construction; these are robust, light, inexpensive, and of limited accuracy (0.5-1.0\% moisture).

- Laboratory equipment intended for rapid water content determination on samples taken from the production line. These are meters of moderate accuracy, calibrated for quick measurements of one or more similar materials. This category should be distinguished from equipment using microwave energy for heating and drying samples with simultaneous or subsequent weighing to determine the moisture content. The time required for moisture determination in the latter is about 0.1 to 1 hour, while the calibrated microwave moisture meter can make determinations on 50 to 100 samples per hour.

- Research laboratory instruments designed for several investigations of materials containing water, which should provide high accuracy and versatility without operational simplicity, reading facility, data processing, etc. It may be noted that scientific interest shifts from materials in bulk to particles and eventually to the structure of particles for better understanding of such phenomena as water binding mechanisms in the material structure and drying processes. In this category, precise instruments for the measurement of dielectric properties can be listed, such as impedance meters, dielectrometers, network analyzers, and other modern, automatic, computerized instruments.

- Industrial on-line equipment for continuous operation in manual or automatic process control. In such equipment, unauthorized adjustments or corrections are not allowed, and an output signal proportional to the average moisture content in the material is recorded as well as used for process control. Here high reliability and stability of the instrument are the most important characteristics. Self-checking and calibrating systems, together with easy fault detection, are important assets, because every breakdown means stoppage of the technological process, which is costly and unpleasant. On the other hand, accuracy of measurement is often difficult to determine, and it is supplanted by repeatability and stability. Usually the cost of the instrumentation is not important, because it can be repaid in a few months by significant savings in energy cost and improved product quality.

The design of different types of moisture meters requires individual considerations, even when they are to be used for the same material. In any particular application, such factors as the specific properties and structure of material, peculiar requirements of location, measuring conditions, display of results, cost, expected number of instruments to be sold for similar purposes, required complexity, reliability, and precision should be carefully taken into consideration.

For simple, reliable, and inexpensive portable moisture meters which could be used in routine measurements by inexperienced personnel, often under field conditions, microwave meters offer significant advantages over simple chemical testers (acetylene or toluene based) in terms of the time required to finalize the test. They also would offer advantages over simple resistive and dielectric meters in terms of accuracy and repeatability if they were competitive in price. The static character of the measurement allows preparation of the sample under test so that effects of density changes can be greatly limited. Also, the temperature of the sample should be taken into account during calibration. Simple microprocessors would be helpful in these applications.

Laboratory microwave moisture meters should also be competitive with electronic moisture content meters based on measurements of conductivity or permittivity of wet materials in the RF range. The latter, however, have an established position in the market. They operate successfully in many branches of industry, fulfilling the majority of the requirements imposed. In some cases, e.g., in the trade of grain and textiles, their application is recommended by national and international regulations. Because of mass production, their prices are lower than those of existing microwave meters. A well-designed two parameter microwave meter should prove superior to these meters, for reasons already stated and because no weighing of the material is required to provide information on the material density.

In laboratory research work, all conventional microwave measuring instruments and techniques operating in the frequency and time domains can be successfully applied. Modern automatic network analyzers render 

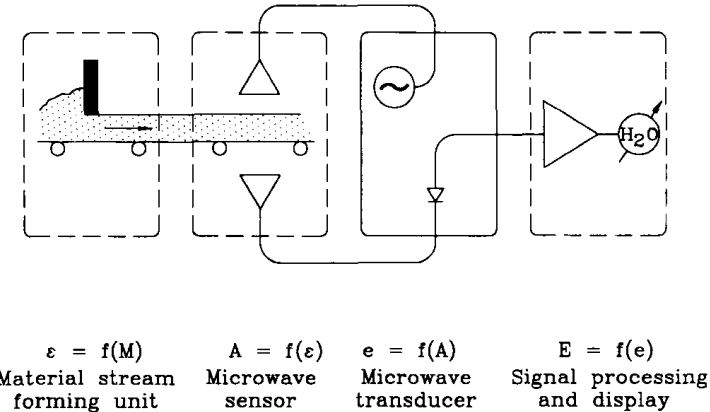

Fig. 1. Block diagram of the microwave moisture content meter

much help in research on, for example, dielectric mixtures containing water, the physics of water binding, and the mechanisms of material drying. The choice of a particular measuring technique results from the physical structure of the material to be measured, convenience, and the customs of the investigator rather than from any general rule.

During the last several years, microwave moisture meters have been successfully applied in continuous on-line moisture monitoring and control systems in several branches of industry [5]. The high environmental tolerance of microwave instruments, resulting from the design of components for military or space applications, enables these instruments to function well in many industrial environments.

A microwave moisture content measuring system may be considered as a chain of information transducers connected as shown in Fig. 1. Such a system, from a metrological point of view, may be divided into the following units:

1) Material Stream-Forming Unit: This prevents variation of the permittivity of the material, being a mixture of constant water content, that results from changes in water-material binding, chemical reactions in the mixture containing water, its temperature, and the density of the mixture. Mechanical means such as vibrators, rollers, and scrapers, are used to provide proper measuring conditions in the sensor area, i.e., the laminar flow of material, specified layer thickness of uniform density or flat material surface. The performance of the unit may be described by the general function $\epsilon=f(M)$, relating material moisture content and its permittivity.

2) Microwave Sensor: It is here that the interaction of the electromagnetic wave with the wet material takes place. The sensor should provide a given microwave signal for a given permittivity of the test material. Possible output signal variations come from fluctuations of the layer thickness, displacement of the material within the measuring space, and nonuniformity of the microwave field. The relationship between a microwave parameter of the material, e.g., attenuation, $A$, and the dielectric permittivity of the mixture may be expressed in a general form as $A=f(\epsilon)$. Many forms of radiating elements in

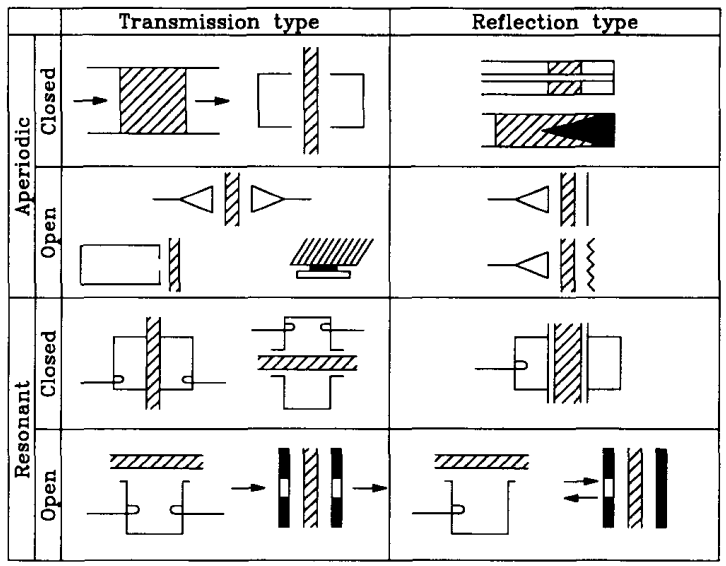

Fig. 2. Classification of microwave sensors being used for moisture content measurement.

waveguide, coaxial-line, or stripline configurations can be used as microwave sensors. They may be divided into resonant and aperiodic groups [11]-[13], into open and closed structure, and into reflection and transmission types. A classification and some examples of microwave sensors are shown in Fig. 2.

3) Microwave Transducer: This converts the microwave signal, proportional to moisture content, into an electrical signal. It consists of a microwave oscillator, a transmission system, and a detector, with the sensor being connected into the transmission path. There are many parameters being used as a measure of moisture in microwave moisture meters, for example, the energy absorbed in wet material, the phase shift introduced by the material, the reflection coefficient from wet material surface, or the resonant cavity detuning caused by the introduction of a wet sample. Each of these parameters can be precisely measured in conventional microwave circuits operating on the basis of deflection, differential, or null methods, including substitution, comparison, and compensation methods. Variation of an electric output signal $e$ at a constant value of microwave parameter $A$ may result from changes of losses and mismatches in microwave components, from fluctuation of the output power of the oscillator, and from conversion loss of the detector. All these may affect the transducer operation function $e=$ $f(A)$ and cause errors in the moisture content measurement.

4) Signal Processing Unit: This contains power supplies, amplifiers of the electric input signal $e$, microprocessors, and means allowing transformation of the moisture meter output signal $E$ into analog or digital indicators or recorders and to the automatic control system. A general relation between the output signal $E$ and its input value $e$ may be expressed in form of $E=f(e)$. The performance of this unit is affected by incidental changes in the sensitivity of electronic components, fluctuations, electronic noise, and interference. In simple 
meters in the past, square-law detectors were most frequently used. Recently, heterodyne or homodyne detectors have been used [14], [15]. The unit should ensure high sensitivity and resolution at permissible instability of the $e / E$ conversion function resulting from component aging and environmental effects (short- and long-term drifts). Very often small fluctuations of moisture content in a dynamic technological process are integrated in time, because, from the user's point of view, it is much more important to monitor the average moisture content than the instantaneous value, which often fluctuates widely because of the process variables.

A general rule in moisture meter design should be that the slope of the main conversion function $M / E$ be high and stable and all unwanted, masking side effects be kept small and constant. Each meter should be designed bearing its application in mind, as well as the environmental conditions under which it is expected to operate. Experience shows that successful applications are those for which the meter provides a profit to the user (in terms of improving product quality, production capacity, cost reduction, process automation, etc.), rather than those used in settling labor disputes, competing with other production divisions in a factory, or enforcing laws and regulations.

\section{Experimental Data}

Some experimental data are included here to show the general nature of the principles mentioned. First, experiments for moisture content determination in single soybean seeds are presented, and then moisture content measurements in a flowing stream of wheat are discussed. Although these examples are for agricultural products, microwave moisture content meters are by no means limited to such products. Similar results could be obtained in preparation for microwave monitoring of moisture content in sand in a glass factory, in crushed coal and coal dust in a coal processing plant, in artificial fertilizers, in pharmaceutical granules and powders, and in many other materials of various forms.

\section{A. Single Soybean Seeds}

Soybeans are seeds of nearly spherical shape, and the ratios of the major and minor diameters of the lot tested ranged from 1.2 to 1.36. Major diameters ranged from 6.5 to $7.5 \mathrm{~mm}$. Eighteen seeds were selected for microwave cavity measurements to establish a calibration [16]. The initial moisture content was about $16 \%$ and the seeds were permitted to dry under room conditions for various time intervals and then were stored in glass vials for several days to obtain more uniform moisture distribution within the seed. They were weighed after each cavity measurement to an accuracy of $0.2 \mathrm{mg}$ and finally were dried in an oven to determine the dry weight. The moisture content of each seed was calculated for each measurement to complete the calibration. The moisture content ranged from $8 \%$ to $16 \%$.
A rectangular waveguide resonant cavity was used as a measuring sensor. The cavity consisted of a section of standard WR-187 rectangular waveguide $203 \mathrm{~mm}$ long, coupled to the external waveguides through two identical apertures of $13 \mathrm{~mm}$ in diameter. The cavity operated in the $H_{107}$ mode at $6017.4 \mathrm{MHz}$, with $Q_{L 0}=830$. Individual soybean seeds were attached to thin silk threads, permitting them to be suspended in the center of the cavity. The cavity was located between two waveguide-to-coaxial transitions, which allowed it to be connected to an automatic network analyzer calibrated in the transmission mode at 401 discrete frequencies with a range of $15 \mathrm{MHz}$ spanning the resonant frequency. The shift of the resonant frequency and transmission factor of the cavity were measured with a soybean seed loading the cavity. The shift of resonant frequency is denoted as $\Delta F=f_{0}-f_{s}$, where the subscripts 0 and $s$ refer to the empty cavity and to the cavity loaded with a seed, respectively. Energy dissipated in the seed can be expressed in terms of changes in the cavity $Q$ factor:

$$
\frac{1}{Q_{L s}}-\frac{1}{Q_{L 0}}=\frac{1}{Q_{L 0}}\left(\frac{Q_{L 0}}{Q_{L s}}-1\right)=\frac{1}{Q_{L 0}}\left(\frac{V_{0}}{V_{s}}-1\right)=\frac{\Delta T}{Q_{L 0}}
$$

where $Q_{L}$ is the $Q$ factor of the cavity, and $V$ is the voltage transmission coefficient at resonance, which can be easily measured. The transmission factor $\Delta T=10^{k}-1$, where $k=0.05\left(S_{210}-S_{21 s}\right)$, with $S_{21}$ being the voltage transmission coefficient at resonance, expressed in decibels.

Experimental results for the 18 seeds measured at six hydration levels each are shown in Fig. 3. The shift of resonant frequency, $\Delta F$, as a function of the water content, $w_{w}$, is presented in Fig. 3(a), while in Fig. 3(b) the transmission factor, $\Delta T$, versus the water content is shown. Two linear equations fit the experimental results with a high statistical significance:

$\begin{array}{lll}\Delta F=-0.675+0.28617 w_{w}+0.0495 w_{d}, & r=0.9966 \\ \Delta T=-0.02633+0.02226 w_{w}-0.000656 w_{d}, & r=0.9946\end{array}$

where $r$ is the correlation coefficient. According to the theoretical considerations, (1), (3), and (5), the moisture content, in percent, of an individual soybean seed may be expressed as

$$
M=\frac{X+18.9(4+Y)}{0.349 X-(3.61-Y)}
$$

where $X=\Delta F / \Delta T$ and $Y=0.141 / \Delta T$, without regard to the seed dimensions or shape.

To test the validity of the calibration equation (6), another 55 soybeans of moisture content ranging from $7 \%$ to $15 \%$ were measured in the cavity. Their exact moisture contents were determined by the standard oven method and compared with those calculated from (6). The mean value of difference (bias) was $0.06 \%$ moisture, and the standard deviation of the difference (SEP, standard error of performance) was $0.46 \%$ moisture. Thus, with the microwave resonator method, the moisture content of 


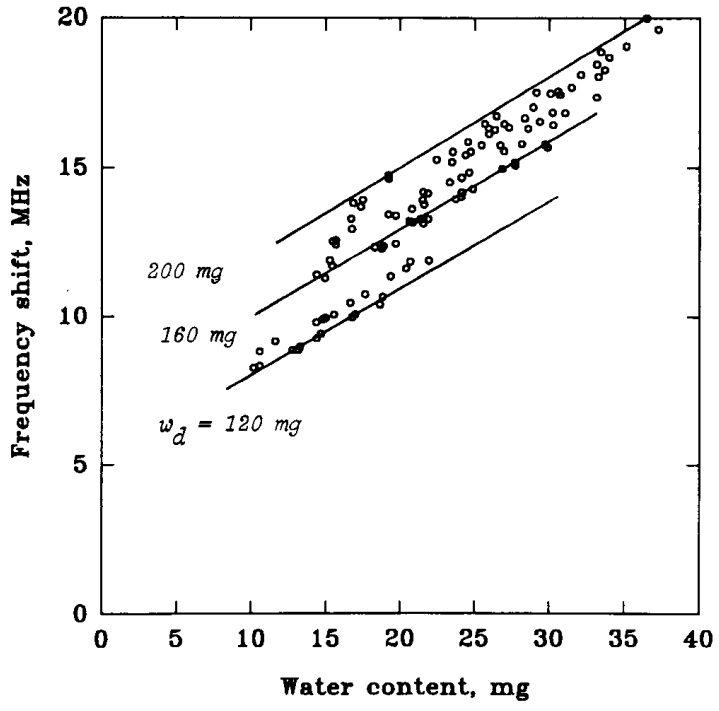

(a)

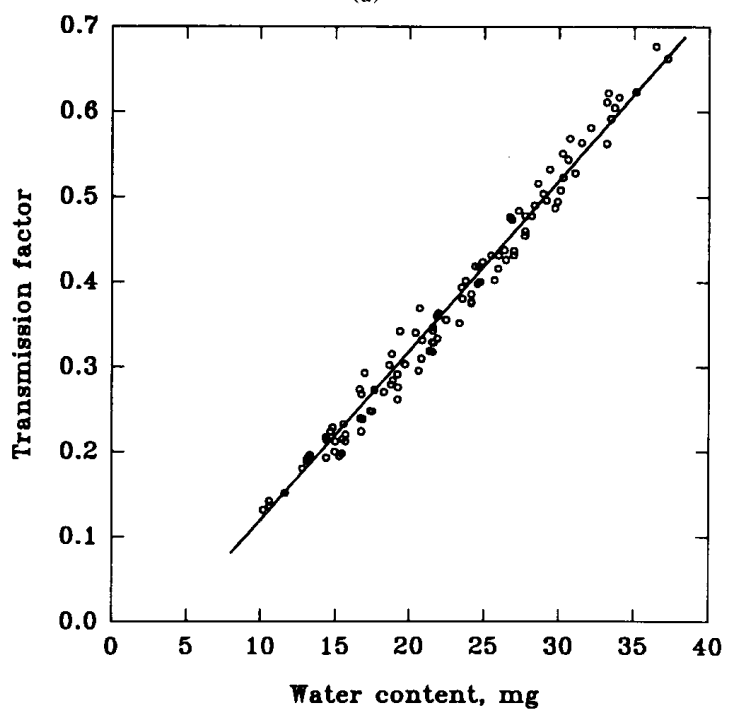

(b)

Fig. 3. (a) Resonant frequency shift and (b) Transmission factor, as functions of water content in single soybean seeds located in the resonant cavity.

soybean seeds in the range from $7 \%$ to $16 \%$ can be determined with an uncertainty of less than $1 \%$ at the 95\% confidence level.

\section{B. Wheat Grain in Bulk}

A standard microwave bridge measuring circuit operating at $9.4 \mathrm{GHz}$ was used to measure the attenuation and phase shift introduced by a wheat sample placed in a dielectric container with a volume of about 3 liters located between two matched horn antennas separated by
$15 \mathrm{~cm}$. The container, $12 \mathrm{~cm}$ in total thickness, was made of 1 -cm-thick Plexiglas ${ }^{1}$ sheet, acting as a half-wavelength impedance transformer. A rotary-vane attenuator and a calibrated waveguide phase shifter were used to precisely balance the bridge, first without and then with the sample between the horn antennas. Samples of wheat were dried for various periods of time under room conditions from $17.5 \%$ to $9.5 \%$ moisture [9]. The moisture content of wheat was determined after each measurement by a standard oven method. The accuracy of this procedure was established to be better than $\pm 0.3 \%$ moisture [17].

Linear relationships between both attenuation and phase shift and the moisture content were observed for wheat samples of various densities (layer of thickness $t=10 \mathrm{~cm}$ ) as shown in Fig. 4. These relationships can be expressed in the form

$$
A=1.79 M-9.08 \text { and } \phi=18.13 M+44.9
$$

where $A$ is the attenuation in decibels and $\phi$ is the phase shift in degrees. It is interesting to note that the relationships between the microwave parameters and the moisture content remain linear when $A$ and $\phi$ in (7) are normalized to the actual bulk density of grain, $\rho$. The following expressions, obtained by least-square regression, describe the experimental data presented in Fig. 5:

$$
\frac{A}{\rho t}=0.293 M-1.936, \quad r=0.991
$$

and

$$
\frac{\phi}{\rho t}=3.558 M+45.32, \quad r=0.989 .
$$

When the attenuation and the phase shift of an electromagnetic wave passing through the layer of wet wheat can be measured simultaneously, by solving both equations (8), one can separate the two unknown variables (moisture content and density) and obtain two independent expressions in the form

$$
\begin{gathered}
M=\frac{154.7+6.61 X}{X-12.1} \\
\rho=\frac{0.293 \phi-3.56 A}{20.17 t}
\end{gathered}
$$

where $X=\phi / A$ and $t$ is the layer thickness in $\mathrm{cm}$. It is evident from (9) that the value of moisture content does not depend upon the thickness of the material layer or upon the density of the material. The value of the actual density determined from (10) may be further used for correction of the reading. The uncertainty in moisture content determination using the calibration equation (9) may be expressed as

$$
\Delta M=-\frac{20.17 X}{(0.293 X-3.56)^{2}}\left(\frac{\Delta A}{A}+\frac{\Delta \phi}{\phi}\right)
$$

\footnotetext{
${ }^{1}$ Mention of company or trade names is for purpose of description only and does not imply endorsement by the U.S. Department of
} Agriculture. 


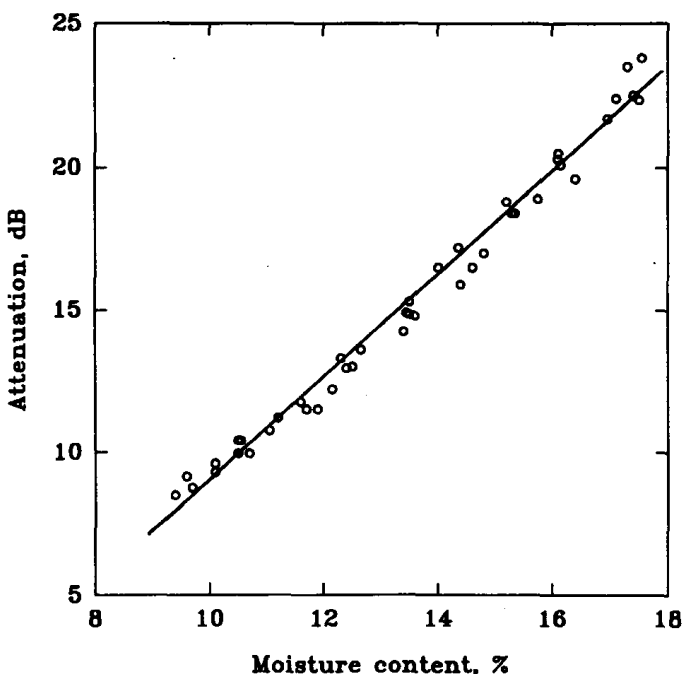

(a)

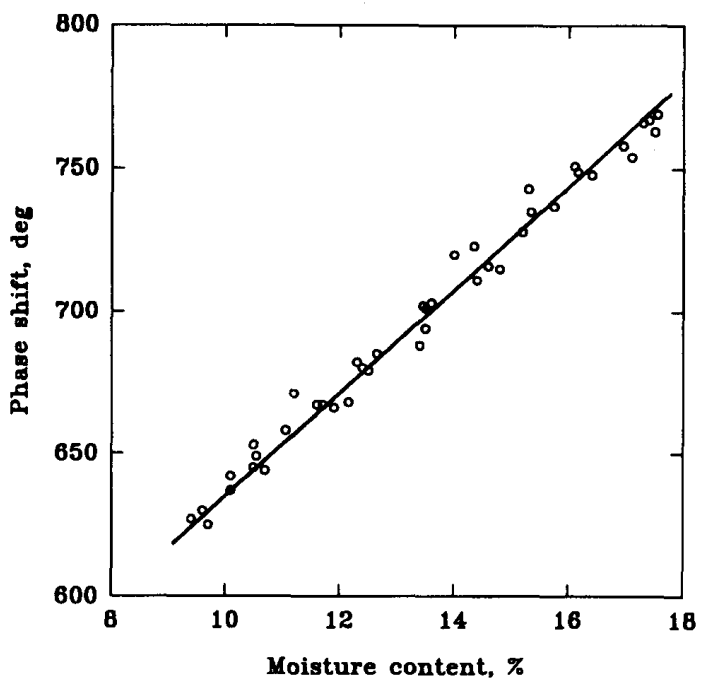

(b)

Fig. 4. (a) Attenuation and (b) phase shift in wheat of various densities and thickness of $10 \mathrm{~cm}$, as functions of grain moisture content.

where $\Delta A$ and $\Delta \phi$ are the uncertainties in the measurement of the attenuation and the phase shift, respectively. The uncertainty in moisture content measurement (11) is also independent of the density of the material under test.

The validity of the calibration equation for wheat was tested using 176 experimental data points for wheat, which were not used in developing the equation. The estimated moisture content was calculated from (9) for all of those experimental points and compared with the corresponding moisture content determined for the sample by the standard oven method. The mean value of differences is $0.02 \%$ moisture, and the standard deviation of differences is $0.17 \%$ moisture. Thus, the moisture con-

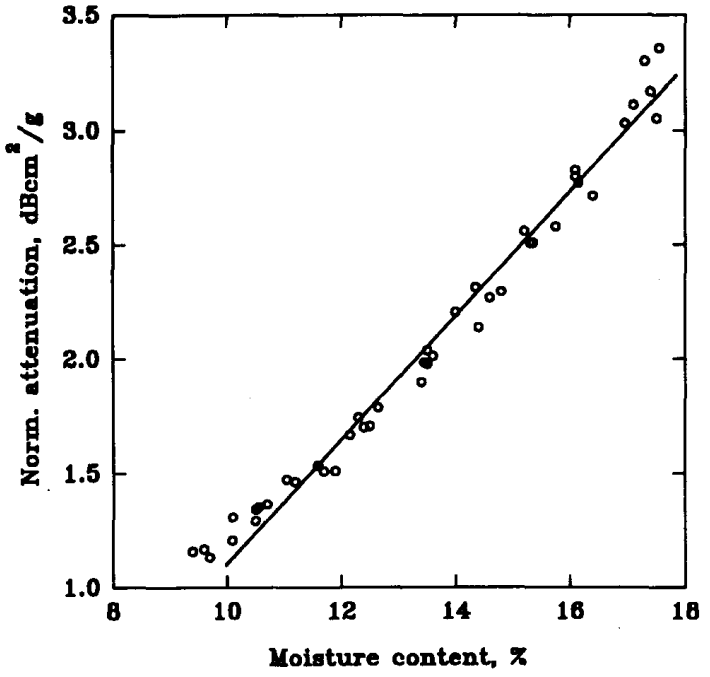

(a)

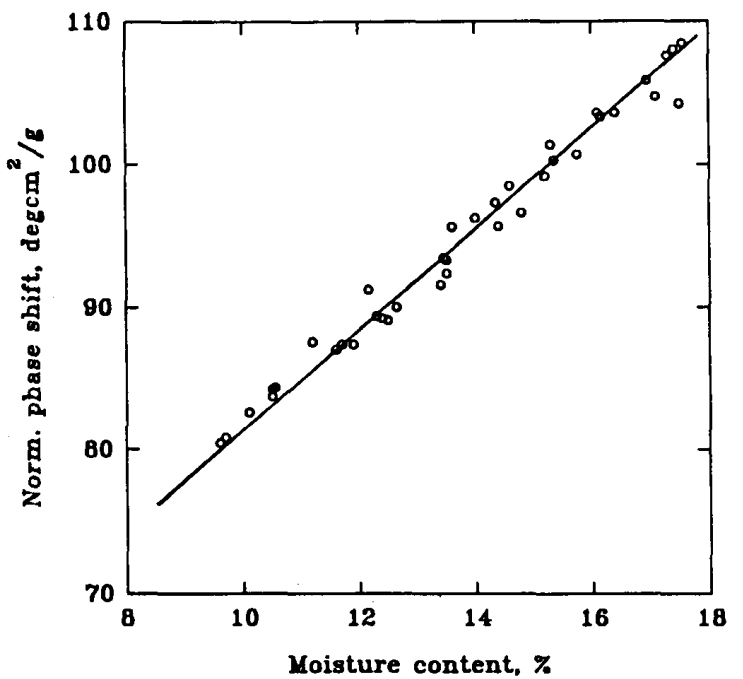

(b)

Fig. 5. (a) Attenuation and (b) phase shift in wheat normalized to the layer thickness and actual grain density, as functions of grain moisture content.

tent of wheat in the range from $9.5 \%$ to $17.5 \%$ can be determined with an uncertainty of $0.33 \%$, at the $95 \%$ confidence level, regardless of the $5 \%$ to $8 \%$ grain density variation, simply by using (9). One should bear in mind that the uncertainty related to the standard oven method itself could be as high as $0.3 \%$ moisture.

The examples presented were for static measurements carried out under laboratory conditions with custom laboratory equipment. There is, however, no contact required between the sample of the material under test and the measuring setup, so it is easy to imagine that the sample material could be flowing through the container or that 
the single seed could be measured during free fall through the cavity. The transition from laboratory experiment to continuous process monitoring in industrial conditions, however, is much more complex and labor consuming than this statement might indicate. Many promising projects in the past have failed because laboratory measuring instruments were used in industrial environments, causing problems and creating disappointment in the method and discouragment among the researchers.

\section{CONClusions}

Rapid development of automation in industrial processes related to energy-saving programs creates a growing need for improved sensors for process monitoring and control. Microwave radiation can be a superior tool for many applications related to moisture content measurements. The development of increasingly reliable and efficient solid-state microwave sources, integrated components, and systems has stimulated new interest in industrial and laboratory applications of microwave moisture meters by making them more competitive in price with conventional measuring equipment. General improvement should be provided in the future in the effectiveness of the theoretical predictions of the dielectric properties of wet materials, in the synthesis of the measuring systems, in meter calibration procedures, and in error analysis (in theory and in practice), allowing better design of the equipment, cheaper production, and wider application of microwave moisture content meters.

The main problems in the development of a new microwave meter or in the adaptation of an existing one to a new material are the choice of the optimal sensor to ensure effective coupling of the microwave energy into the material, and appropriate conditioning of the material to meet the constant density requirement. The latter problem may be overcome by simultaneous measurement of two parameters of the electromagnetic wave interacting with the material [17], [18]. This requires a slight complication of the measuring system and signal processing unit but achieves a considerable cost reduction by eliminating the need for an expensive and complex material stream processing unit. Further development of microwave moisture meters requires the development of better microwave sensors and better measuring systems. One of the purposes of this paper in the special issue is to draw the attention of microwave engineers to problems of nondestructive testing of materials and to stimulate their imagination and ingenuity in providing original solutions to these problems.

\section{REFERENCES}

[1] W. Schilz and B. Schiek, "Microwave systems for industrial measurements," in Advances in Electronics and Electron Physics, vol. 55. New York: Academic Press, 1981, pp. 309-381.

[2] M. A. Stuchly and S. S. Stuchly, "Industrial, scientific, medical and domestic applications of microwaves," Proc. Inst. Elec. Eng., vol. 130 , pt. A, no. 8, pp. 467-503, 1983.
[3] A. Kraszewski, "Microwave instrumentation for moisture content measurement," J. Microwace Power, vol. 8 , nos. 3 and 4 , pp. $323-336,1973$.

[4] V. K. Benzar, Moisture Measurements at UHF. Minsk, USSR: Vysshaya Shkola, 1974 (in Russian).

[5] A. Kraszewski, "Microwave aquametry-A review," J. Microwate Power, vol. 15, no. 4, pp. 209-220, 1980.

[6] S. O. Nelson, "Use of electrical properties for grain-moisture measurement," J. Microwave Power, vol. 12, no. 1, pp. 67-72, 1977.

[7] A. Kraszewski, "Microwave aquametry-Bibliography 1980-1989," J. Microwave Power, vol. 26, 1991.

[8] S. O. Nelson and A. Kraszewski, "Grain moisture content determination by microwave measurements," Trans. Amer. Soc. Agric. Engrs., vol. 33, no. 4, pp. 1303-1307, 1990.

[9] A. Kraszewski, "Microwave monitoring of moisture content in grain -Further considerations," J. Microwace Power, vol. 23, no. 4, pp. 236-246, 1988

[10] A. Kraszewski and S. Kulinski, "An improved microwave method of moisture content measurement and control," IEEE Trans. Ind. Electron. Contr. Instrum., vol. IECI-23, no. 4, pp. 364-370, 1976.

[11] F. E. Gardiol, "Open-ended waveguides: Principles and applications," in Adcances in Electronics and Electron Physics, vol. 63. New York: Academic Press, pp. 139-187.

[12] R. W. P. King and G. S. Smith, Antennas in Matter. Cambridge, MA: MIT Press, 1981.

[13] E. Nyfors and P. Vainikainen, Industrial Microwate Sensors. Norwood, MA: Artech House, 1989

[14] R. J. King, Microwale Homodyne Systems. London, UK: Peter Peregrinus, 1978.

[15] J. Kalinski, "A chopped sub-carrier method of simultaneous attenuation and phase shift measurement under industrial conditions," IEEE Trans. Ind. Electron Contr. Instrum., vol. IECI-28, no. 3, pp. 201-209, 1981.

[16] A. W. Kraszewski, T.-S. You, and S. O. Nelson, "Microwave resonator technique for moisture content determination in single soybean seeds," IEEE Trans. Instrum. Meas., vol. 38, no. 1, pp. $79-84,1989$.

[17] A. Kraszewski, S. Kulinski and Z. Stosio, "A preliminary study on microwave monitoring of moisture content in wheat," $J$. Microwate Power, vol. 12, no. 3, pp. 241-251, 1977.

[18] S. D. Powell, B. D. McLendon, S. O. Nelson, A. Kraszewski, and J. M. Allison, "Use of a density-independent function and microwave measurement system for grain moisture measurement," Trans. Amer. Soc. Agric. Engrs., vol. 31, no. 6, pp. 1875-1881, 1988.

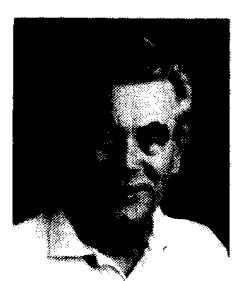

Andrzej W. Kraszewski (M'89) was born in Poznan, Poland, on April 22, 1933. He received the M.Sc. degree in electrical engineering from the Technical University of Warsaw, Warsaw, Poland, in 1958 and the D.Sc. degree in technical sciences from the Polish Academy of Sciences (PAN), Warsaw, in 1973.

In 1953 he joined the Telecommunication Institute (PIT) in Warsaw, Poland, where he did research and development work on microwave systems and components. In 1963 he joined UNIPAN Scientific Instruments, a subsidiary of the Polish Academy of Sciences, as Head of the Microwave Laboratory. In 1972 he became the Manager of the Microwave Department of WILMER Instruments and Measurements, a subsidiary of the Polish Academy of Sciences in Warsaw, where he codeveloped microwave instruments for moisture content measurements and control. Beginning in November 1980, he was a Visiting Professor at the University of Ottawa, Ontario, Canada, where he did research on RF and microwave dosimetry. In January 1987 he joined the Richard B. Russell Agricultural Research Center (U.S. Department of Agriculture) in Athens, Georgia, where he is involved in research on plant structure and composition using electromagnetic fields.

Dr. Kraszewski is a member of the International Microwave Power Institute, the Materials Research Society, and the Polish Electricians Association. He is the author of several books on microwave theory and techniques, has published more than 100 technical papers on the subject, and holds 18 patents. He has received several professional awards, among them the State Prize in Science in Poland in 1980. 